\title{
Dynamic Interactions of Epidermal Collagen XVII with the Extracellular Matrix
}

\section{Laminin 332 as a Major Binding Partner}

\author{
Wataru Nishie, ${ }^{* \dagger}$ Dimitra Kiritsi, ${ }^{*}$ \\ Alexander Nyström, ${ }^{*}$ Silke C. Hofmann, ${ }^{*}$ \\ and Leena Bruckner-Tuderman ${ }^{\star \ddagger}$ \\ From the Department of Dermatology,* University Medical Center \\ Freiburg, and the Freiburg Institute for Advanced Studies School \\ of Life Sciences-LifeNet, ${ }^{\ddagger}$ University of Freiburg, Freiburg, \\ Germany; and the Department of Dermatology, ${ }^{\dagger}$ Hokkaido \\ University Graduate School of Medicine, Sapporo, Japan
}

Transmembrane collagen XVII, a major component of the hemidesmosomes, is crucial for stable adhesion of the epidermis and dermis in the skin, and its dysfunction results in blistering diseases. The ectodomain of collagen XVII (Ecto-ColXVII) is constitutively shed from the cell surface, but its binding partner(s) in the extracellular matrix (ECM) and the physiologic roles of the ligand interactions remain elusive. Herein, we used a new cleavage site-specific antibody to address the dynamics of collagen XVII shedding and the interactions of Ecto-ColXVII with the ECM. Ecto-ColXVII was present in the migration tracks of primary human keratinocytes and co-localized with laminin 332. The presence of this laminin, but also of collagen IV and Matrigel, in the ECM enhanced shedding and incorporation of Ecto-ColXVII into the matrix. Laminin 332 is a major, but not exclusive, interaction partner in vivo because Ecto-ColXVII deposited in the ECM of laminin 332-deficient keratinocytes was drastically reduced, but Ecto-ColXVII was present in laminin 332-negative human skin. Expression of collagen XVII deletion mutants in HEK 293 cells identified the C-terminal ectodomain stretch Ser ${ }^{978}$. Pro $^{1497}$ as necessary for ECM binding. Taken together, migrating keratinocytes shed the Ecto-ColXVII, and this dynamically binds via its C-terminal domain to distinct partners in the ECM. (AmJ Pathol 2011, 179:829-837; DOI: 10.1016/j.ajpath.2011.04.019)

The extracellular matrix (ECM) is not merely a supporting scaffold for tissue and organ architecture. The ECM can bind and release growth factors, which regulate many biologic processes and cell functions, including migration, differentiation, and proliferation. ${ }^{1}$ Interaction of cells with ECM proteins is usually mediated by cell surface receptors of the integrin or syndecan families, ${ }^{1-3}$ but other tissue-specific receptors, such as collagen XVII, also exist. ${ }^{4}$ A major component of epithelial basement membranes, which represent highly specialized ECM, is laminin 332, and its interactions with keratinocyte integrins $\alpha 3 \beta 1$ and $\alpha 6 \beta 4$ guide cell migration, differentiation, and proliferation. ${ }^{5,6}$

In basal keratinocytes of the epidermis, the adhesion receptors are embedded in larger protein complexes, the hemidesmosomes (HDs), which can be observed as electron-dense ultrastructural components facing the basement membrane. ${ }^{7}$ HDs play an important role in cell attachment to the epidermal basement membrane, as shown indirectly by abnormalities of HDs in congenital and acquired blistering diseases, such as junctional epidermolysis bullosa and pemphigoid diseases. ${ }^{8,9}$ In addition to integrin $\alpha 6 \beta 4$ and laminin 332, HDs contain BP230 and plectin as intracellular components and CD151 and collagen XVII as transmembrane components. ${ }^{7}$ Elucidation of biologic consequences of mutations in the genes encoding for these proteins has uncovered both laminin 332 and collagen XVII as vital players in cell-ECM interactions. ${ }^{10,11}$ However, the interaction mechanisms of collagen XVII with the ECM remain poorly understood.

Collagen XVII is a type II integral transmembrane protein with an intracytoplasmic amino terminus and an extracellular carboxyl terminus, which consists of 15 collag-

Supported in part by a Grant-in-Aid for Young Scientists A (20689021 to W.N.) and by the Deutsche Forschungsgemeinschaft (grant SFB-850/B6 to L.B.-T.).

Accepted for publication April 14, 2011.

Address reprint requests to Wataru Nishie, M.D., Ph.D., Department of Dermatology, Hokkaido University Graduate School of Medicine, N15, W7, Sapporo 060-8638, Japan; or Leena Bruckner-Tuderman, M.D., Ph.D., Department of Dermatology, University Medical Center Freiburg, Hauptstr. 7, D-79104, Germany. E-mail: nishie@med.hokudai.ac.jp or brucknertuderman@uniklinik-freiburg.de. 
enous and 16 noncollagenous subdomains. ${ }^{4,12,13}$ The cytoplasmic domain of collagen XVII has been reported to interact with BP230, plectin, and integrin $\beta 4$; all of these interactions are important for stabilization of the HDs. ${ }^{14,15}$ Thus, collagen XVII is thought to be a vital cell surface receptor that links the cytoplasmic structural components with the ECM. Extracellular binding between the noncollagenous 16th domain of collagen XVII and $\alpha 6$ integrin has also been proposed; the $\alpha 6$ integrin binding site in the collagen molecule was presumed to be within the stretch $\mathrm{Arg}^{506}-\| \mathrm{e}^{519} .{ }^{16}$ This would be juxtamembranous and upstream of the recently disclosed major sheddase cleavage sites. ${ }^{17}$ Thus, the interaction of the shed ectodomain of collagen XVII (Ecto-ColXVII) with $\alpha 6 \beta 4$ integrin still remains elusive. A study of $\mathrm{HaCaT}$ keratinocytes proposed a p38MAPK-dependent functional relationship between collagen XVII and collagen IV and/or other components in Matrigel (BD Biosciences, Franklin Lakes, NJ) that regulates cell attachment and migration. ${ }^{18}$ Furthermore, an in vitro solid-phase protein-protein binding assay using recombinant collagen XVII fragments and laminin 332 indicated an affinity of collagen XVII carboxyl terminus with laminin 332. ${ }^{19}$ Despite the limitations of such assays, the suggestion of a partnership between these two HD components is intriguing and has high pathophysiologic significance because aberrant expression of laminin 332 and collagen XVII has been suggested to be involved in the invasion and proliferation of various cancer cells. ${ }^{6,20}$

In this context, it is important to note that two different physiologic forms of collagen XVII exist: a full-length transmembrane form and a released ectodomain. Proteinases of the ADAM family cleave the Ecto-CoIXVII within the juxtamembranous noncollagenous 16th $A$ (NC16A) domain, ${ }^{4,17}$ yielding a shorter form of the protein, Ecto-ColXVII, which remains stable in the ECM and is likely to interact with other ECM proteins. Thus, functionally, collagen XVII plays a dual role as a cell surface receptor for ECM proteins and as a matrix component.

The study of context-dependent regulation of collagen XVII shedding has been impeded by a lack of precise tools to visualize and measure ectodomain shedding. This problem has been overcome by a recently generated novel antibody (Ab), HK139, which specifically detects cleaved Ecto-ColXVII. ${ }^{17}$ Herein, we used the Ab HK139 to follow shedding of the Ecto-CoIXVII and its deposition in ECM in normal human keratinocytes (NHKs). We show that these processes are dynamic and that distinct ECM proteins play essential roles in interacting with collagen XVII in keratinocytes in vitro and in human skin in vivo.

\section{Materials and Methods}

\section{Tissue Specimens and Cell Culture}

Skin biopsy specimens were obtained from healthy volunteers and from a female neonate with laminin 332negative Herlitz junctional epidermolysis bullosa after informed consent was provided. The genetic and phenotypic features of patients with Herlitz junctional epi- dermolysis bullosa have been described elsewhere. ${ }^{21}$ This study was approved by the Ethical Committee of the University of Freiburg.

Primary NHKs were isolated from normal and laminin 332-negative skin and were cultured in serum-free keratinocyte growth medium supplemented with bovine pituitary extract and epidermal growth factor (Invitrogen, Carlsbad, CA). Cells up to the fourth passage were used for this study. Immortalized HaCaT keratinocytes, a generous gift from Dr. Norbert E. Fusenig (German Cancer Research Center, Heidelberg, Germany), were cultured under the same conditions. Every 48 hours, freshly prepared ascorbic acid was added to the culture medium to a final concentration of $50 \mu \mathrm{g} / \mathrm{mL}$ to allow hydroxylation of collagens and proper triple helix folding. ${ }^{22,23}$ Flp-in 293 host cells (Invitrogen) and transformed 293 cells were maintained in Dulbecco's modified Eagle's medium (Invitrogen) containing 10\% fetal bovine serum.

For coating coverslips and culture flasks, various ECM proteins, including $50 \mu \mathrm{g} / \mathrm{mL}$ of rat tail collagen I (number 354236, BD Biosciences), $100 \mu \mathrm{g} / \mathrm{mL}$ of mouse collagen IV (number 354233, BD Biosciences), $333 \mu \mathrm{g} / \mathrm{mL}$ of Matrigel, $2 \mu \mathrm{g} / \mathrm{mL}$ of laminin 332 (Oriental Yeast Co., Tokyo, Japan), and $5 \mu \mathrm{g} / \mathrm{mL}$ of mouse laminin 111 (number 354239, BD Biosciences), were used according to the manufacturers' instructions.

For collagenase treatment, NHKs grown on coverslips were incubated for 2 hours at $37^{\circ} \mathrm{C}$ with $100 \mathrm{U} / \mathrm{mL}$ of bacterial, chromatographically purified collagenase form III (WAK-Chemie Medical GmbH, Steinbach, Germany) in a buffer containing $50 \mathrm{mmol} / \mathrm{L}$ Tris- $\mathrm{HCl}$ and $65 \mathrm{mmol} / \mathrm{L}$ $\mathrm{NaCl}(\mathrm{pH}$ 7.4). After careful washing with PBS, the coverslips were subjected to indirect immunofluorescence (IIF) staining.

\section{Generation of HEK 293 Cells Stably Expressing Deletion Mutants of Human Collagen XVII}

Full-length human collagen XVII cDNA [NM_000494; a gift from Dr. Luca Borradori (Department of Dermatology, University of Bern, Switzerland)] was introduced into the Notl site of pcDNA5/FRT (Invitrogen), designated as COL17 cDNA. To generate different deletion mutants, a 572-bp Xbal-digested fragment was first cut out, and the digested plasmid was self-ligated to obtain Xbal-digested COL17 CDNA. Next, PCR reactions using forward primer Clal-F 5'-AGGGGTCATCGATGCTCACT-3' (the Clal digestion site is shown in italics) and different reverse primers, including COL3-R 5'-CGCTCTAGATCAGTCAGTGCCATAGGGACCCC-3' (the Xbal digestion site is shown in italics and the stop codon in bold) to generate the Met ${ }^{1}-\mathrm{Asp}^{1340}$ amino acid fragment of collagen XVII designated as COL3, NC6-R 5'-CGCTCTAGATCATCTGAATTCAGACCCTCGCA-3' (the Xbal digestion site is shown in italics and the stop codon in bold) for Met ${ }^{1}$-Arg ${ }^{1174}$ amino acids designated as NC6, and COL11-R 5'-CGCTCTAGATCAAGGAATGCCAAGAGCCCCTG-3' (the Xbal digestion site is shown in italics and the stop codon in bold) for Met ${ }^{1}$-Pro ${ }^{977}$ amino acids designated as COL11, were performed using human colla- 
gen cDNA as template. PCR fragments digested with Clal and Xbal were introduced into the corresponding digested sites of the COL17 CDNA plasmid. To generate a short fragment spanning amino acids $\mathrm{Met}^{1}-\mathrm{Met}^{816}$, designated as COL15, linker oligonucleotides 5'-CGATGTGACTCGAGT-3' and 5'-CTAGACTCGAGTCACAT-3' (the stop codon is shown in bold) were inserted into the Clal- and Xbal-digested sites of COL17 CDNA. Finally, the 572-bp Xbal-digested fragment was inserted into each plasmid.

To produce another fragment in which sequences from amino acids Pro ${ }^{997}$-Gly ${ }^{1152}$ were deleted, designated as $\Delta$ Pro $^{997}$-Gly ${ }^{1152}$, Bluescript SKII vector (Agilent Technologies, Waldbronn, Germany) was digested with Apal, followed by self-ligation to use a single Apal site, resulting in Apal-pBS. Then, a Clal- and BamHI-digested 1121-bp fragment from COL17 cDNA was inserted into the Clal- and BamHI-digested sites of Apal-pBS. Finally, the modified vector was digested with Apal, followed by self-ligation to obtain $\Delta$ Pro $^{997}$-Gly ${ }^{1152}$.

The DNA sequences of all the vectors were confirmed by direct sequencing. COL17 CDNA, COL15, NC6, COL11, and $\triangle$ Pro $^{997}$-Gly ${ }^{1152}$, and the pcDNA5/FRT empty vector as control, were co-transfected with pOG44 (Invitrogen) into Flp-In 293 host cells; this enables stable and isogenic expression of each deletion mutant of collagen XVII. Stably expressing cells were selected under $200 \mu \mathrm{g} / \mathrm{mL}$ of hygromycine B (Invitrogen) as previously described. ${ }^{17}$

\section{Antibodies}

Abs, including mouse monoclonal Abs NC16A-1 and NC16A-3, directed to distinct epitopes in the NC16A domain of collagen $\mathrm{XVII},{ }^{24}$ were used in a 1:10 dilution for IIF (NC16A-1) and in a 1:2000 dilution for immunoblotting (NC16A-3). The cleavage-site-specific rabbit polyclonal Ab HK139 was prepared as previously described ${ }^{17}$ and was used at a concentration of $10 \mu \mathrm{g} / \mathrm{mL}$ for IIF and 1 $\mu \mathrm{g} / \mathrm{mL}$ for immunoblotting. For detection of laminin 332 by IIF, the monoclonal $\mathrm{Ab} 6 \mathrm{~F} 12$ to the $\beta 3$ chain $^{25}$ was used in a 1:5000 dilution. For the $\alpha 3$ and $\gamma 2$ chains, BM165 (a gift from Dr. Johannes A. Eble, Munster, Germany) and GB3 (a gift from Dr. Guerrino Meneguzzi, Nice, France) were used in a dilution of 1:5000 and 1:200, respectively. For the detection of laminin 332 by immunoblotting, a polyclonal Ab to laminin 332 (ab14509, Abcam Inc., Cambridge, MA) was used in a 1:2000 dilution. As an internal control for immunoblotting, anti- $\beta$-tubulin Ab (ab6046, Abcam Inc.) was used in a 1:2000 dilution. For staining cytoskeletal actin filaments, rhodamine phalloidin (number R415, Invitrogen) was used.

\section{Immunoblotting and IIF}

For immunoblotting, subconfluent NHKs, HaCaT cells, or transformed HEK 293 cells were incubated in serum-free medium for 24 hours before harvest. Then, the cells were lysed for 30 minutes on ice in a buffer containing $1 \%$ Nonidet P-40 (Caledon Laboratories Ltd., Georgetown, ON, Canada), $0.1 \mathrm{~mol} / \mathrm{L} \mathrm{NaCl}, 25 \mathrm{mmol} / \mathrm{L}$ Tris-HCl, pH7.4, $10 \mathrm{mmol} / \mathrm{L}$ EDTA, and $1 \mathrm{mmol} / \mathrm{L}$ Pefabloc (Roche Diag- nostics Deutschland $\mathrm{GmbH}$, Mannheim, Germany). ${ }^{22,23}$ The medium proteins were precipitated with ethanol before further processing. ${ }^{22}$ Preparation of the ECM proteins was performed as described, ${ }^{26}$ with some modifications. Briefly, after washing with PBS, the cell layer was incubated at room temperature with $20 \mathrm{mmol} / \mathrm{L} \mathrm{NH}{ }_{4} \mathrm{OH}$ solution. After all the cells had detached, the ECM was extensively washed with PBS and dissolved in SDSPAGE sample buffer. The samples were boiled for 5 minutes and were separated on SDS-PAGE using $7 \%$ or $4 \%$ to $13 \%$ gradient polyacrylamide gels, followed by transfer onto nitrocellulose membrane. After incubation with primary Abs diluted with $2 \%$ skim milk in Tris-buffered saline overnight at $4^{\circ} \mathrm{C}$, horseradish peroxidaseconjugated secondary Abs in the same buffer were reacted for 1 hour at room temperature. The signals were visualized by ECL Plus (GE Healthcare, Piscataway, NJ), and the intensity of each band was measured using ImageJ software version 1.44 (http://rsbweb.nih.gov/ij, last accessed June 13, 2011).

For IIF, normal and laminin 332-deficient human skin was mounted and snap frozen into optimal cutting temperature compound, and 5- $\mu \mathrm{m}$ cryosections were prepared. Cells cultured on coverslips were fixed with $4 \%$ paraformaldehyde and were permeabilized with $1 \%$ Triton X-100 (Roche Diagnostics GmbH) in PBS. The sections and coverslips were incubated with primary Abs for 1 hour at room temperature, washed with PBS, and detected with Alexa 488/568-conjugated anti-mouse or anti-rabbit IgG (Invitrogen). Mounting medium with DAPI (VECTASHIELD; Vector Laboratories, Burlingame, CA) was used to mount the coverslips. Fluorescent images were obtained using an Axiophot microscope equipped with an AxioCam MRc digital camera or an LSM 510 META laser scanning confocal microscope (Carl Zeiss Microlmaging $\mathrm{GmbH}$, Jena, Germany).

\section{Results}

\section{Migration Tracks of NHKs Contain the Shed Ecto-ColXVII in the ECM}

IIF staining of cultured NHKs with the Ecto-ColXVII-specific Ab HK139 revealed characteristic "ringlike" or "arclike" staining patterns, combined with numerous small dots around migrating cells (Figure $1 \mathrm{~A}$ ), reflecting dynamic shedding of the Ecto-CoIXVII. The ringlike structures also contained laminin 332 (Figure 1B). Collagen XVII staining was sensitive to collagenase digestion, whereas laminin 332 staining was resistant to it (Figure 1B), indicating that the Ab HK139 detected only a collagenous structure. The Ecto-CoIXVII was visible in the ECM of NHKs approximately 2 to 4 hours after seeding of the cells (Figure $1 \mathrm{C}$ ). When $\mathrm{Ca}^{2+}$ concentration in the medium was increased from $0.09 \mathrm{mmol} / \mathrm{L}$ to $1.2 \mathrm{mmol} / \mathrm{L} 4$ hours after seeding, the cells started to detach, leaving ringlike deposition of the Ecto-CoIXVII in the ECM beneath the cells (Figure 1D). This may suggest that the $\mathrm{Ca}^{2+}$ shift, which is a potent inducer of NHK differentiation, ${ }^{27}$ also induced shedding of the Ecto-CoIXVII, as 
observed in calcium influx-stimulated shedding of the Ecto-CoIXVII. ${ }^{28}$ These results suggest the close relationship between shedding of the Ecto-CoIXVII and migration or differentiation of NHKs.

The Ab HK139 detected the Ecto-ColXVII usually colocalized with laminin 332, indicating their close relationship (Figure 2A). However, some migrating NHKs left tracks containing normal amounts of laminin 332 but little or no Ecto-CoIXVII (Figure 2B). These findings show that the Ecto-CoIXVII mostly co-localizes with laminin 332, but not vice versa, and indicates that laminin 332 can undergo different ligand interactions in a stable ECM.
A

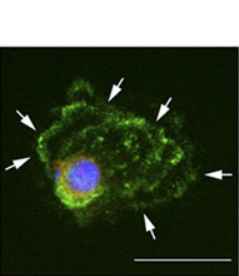

B

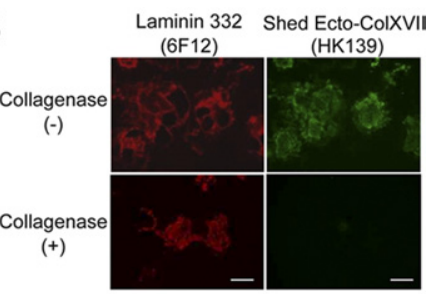

C

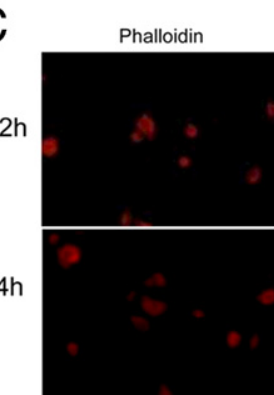

$6 h$

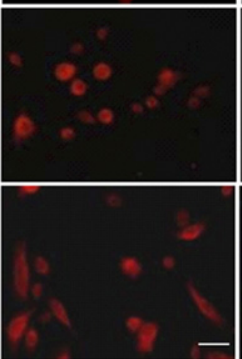

D

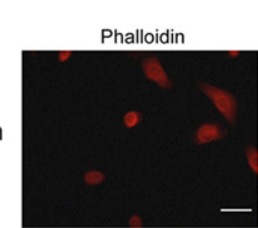

Shed Ecto-ColXVII
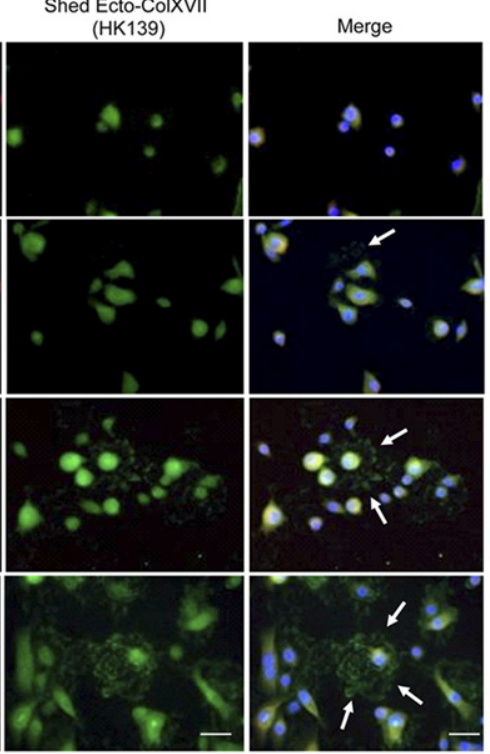

Shed Ecto-ColXVII
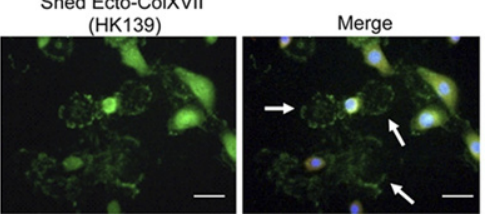

Figure 1. Ecto-ColXVII in the ECM of the migration track of cultured NHKs. A: IIF staining with the Ab HK139 revealed characteristic ringlike or arclike staining patterns that also contained numerous small dots around the migrating cell (arrows). B: The shed Ecto-ColXVII in the ECM was sensitive to collagenase digestion (the Ab HK139; bottom right), whereas laminin 332 (the Ab 6F12, bottom left) persisted. C: The shed Ecto-ColXVII in the ECM of cultured NHKs was detected by the Ab HK139 at 2 to 4 hours after seeding (arrows), and it remained stable for at least 8 hours. D: Thirty minutes after the shift of $\mathrm{Ca}^{2+}$ concentration in culture medium from $0.09 \mathrm{mmol} / \mathrm{L}$ to 1.2 $\mathrm{mmol} / \mathrm{L} 4$ hours after seeding, several NHKs detached, leaving ringlike deposition of Ecto-ColXVII in the ECM (arrows). Red, rhodamine phalloidin in $\mathbf{A}, \mathbf{C}$, and $\mathbf{D}$ and laminin 332 (the Ab 6F12) in B; blue, DAPI. Scale bars = $40 \mu \mathrm{m}$.
A

Laminin $332(6 F 12)$

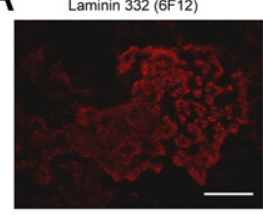

Shed Ecto-CoIXVII (HK139)
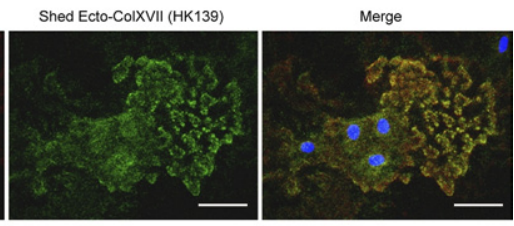

B

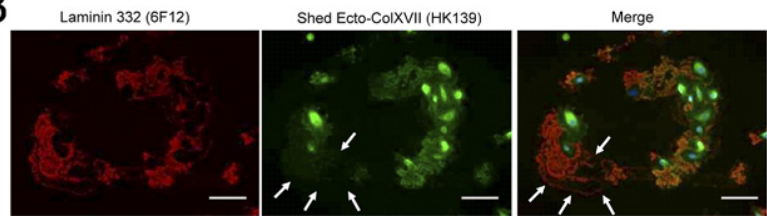

Figure 2. In NHK conglomerates, the shed Ecto-ColXVII usually co-localizes with laminin 332, but not vice versa. A: Confocal microscopy revealed co-localization of shed Ecto-ColXVII (the Ab HK139) with laminin 332 (the Ab 6F12). B: In contrast, some migrating NHKs left tracks containing laminin 332 but very little or no Ecto-ColXVII (arrows). These findings indicate the distinctive and unproved forms of migration in NHKs. Blue, DAPI. Scale bars $=40 \mu \mathrm{m}$.

\section{HaCaT Keratinocytes Deposit Less ECM}

Immortalized HaCaT keratinocytes are commonly used in in vitro experiments to substitute for NHKs. Herein, we compared the ECM produced by NHKs and HaCaT keratinocytes for its content of Ecto-CoIXVII and laminin 332. In contrast to NHKs (Figure 2A), the amounts of Ecto-CoIXVII and laminin 332 were strongly reduced in the ECM of migrating $\mathrm{HaCaT}$ keratinocytes, as assessed by IIF (Figure 3A). Immunoblotting showed that HaCaT cells deposited less Ecto-ColXVII in the ECM than did NHKs (Figure 3B). Mean intensity of the HaCaT keratinocyte-derived bands was $38 \%(n=3)$ of that of NHK-derived bands. Ecto-CoIXVII in culture medium of $\mathrm{HaCaT}$ keratinocytes was also reduced, but to a lesser

A
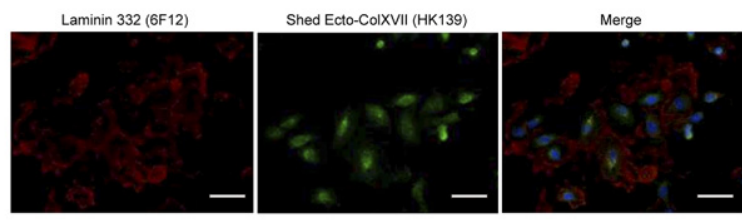

B

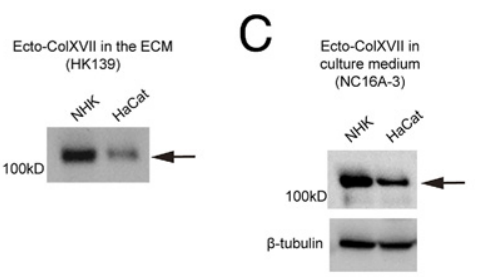

D

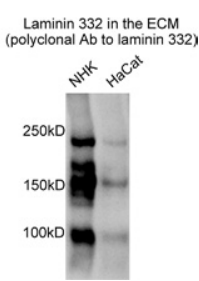

Figure 3. The ECM of immortalized HaCaT keratinocytes differs from that of NHKs. A: The amounts of the shed Ecto-ColXVII (the Ab HK139; green) and laminin 332 (the $\mathrm{Ab} 6 \mathrm{~F} 12$; red) in the ECM of migrating HaCaT keratinocytes was significantly reduced compared with that in the NHKs seen in Figure 2. B: Immunoblotting revealed reduced amounts of Ecto-ColXVII in the ECM of HaCaT cells compared with NHKs. Mean intensity of the HaCaT bands was approximately $38 \%(n=3)$ of that of NHKs. C: The shed Ecto-ColXVII in culture medium was not reduced as strongly as in the ECM, as detected by the Ab HK139. Mean intensity of the HaCaT bands was approximately $80 \%$ $(n=3)$ of that of NHK bands. D: Polyclonal Ab to laminin 332 revealed significantly reduced amounts of laminin 332 chains in the ECM of HaCaT keratinocytes compared with NHKs. Blue, DAPI (A). Scale bars $=40 \mu \mathrm{m}$. 
extent. Mean intensity of the HaCaT medium-derived bands was $80 \%(n=3)$ of that of NHK medium-derived bands (Figure $3 \mathrm{C}$ ). These results demonstrate that $\mathrm{HaCaT}$ cells synthesize and secrete less collagen $\mathrm{XVII}$ than do NHKs but also that the incorporation of Ecto-CoIXVII into the ECM is different from that into NHKs, at least in quantitative terms in that in $\mathrm{HaCaT}$ cell cultures more Ecto-ColXVII remained soluble in the medium. This may reflect reduced laminin 332 and altered composition of the ECM of HaCaT cells (Figure 3D). Thus, these experiments disclosed considerable differences in expression and matrix incorporation of ECM proteins between NHKs and immortalized HaCaT keratinocytes.

\section{Deposition of Ecto-CoIXVII Requires Specific Ligands in the ECM}

To depict the influence of different ECM proteins on shedding of collagen XVII and deposition of the Ecto-CoIXVII in the ECM, HEK 293 cells were transfected with collagen XVII cDNA. When cultured on plastic, these cells do not deposit significant amounts of Ecto-CoIXVII in the ECM (Figure 4). Thus, this cell line is suitable for assessing the role of different ECM proteins, which can interact with collagen XVII and support deposition of the Ecto-ColXVII in the ECM. When the cells were cultured on plastic coated with collagen IV, Matrigel, or laminin 332, the amount of Ecto-ColXVII in the ECM was significantly increased (Figure 4A). Matrigel, which is derived from Engelbreth-Holm-Swarm tumor cells, contains several ECM

\section{A}

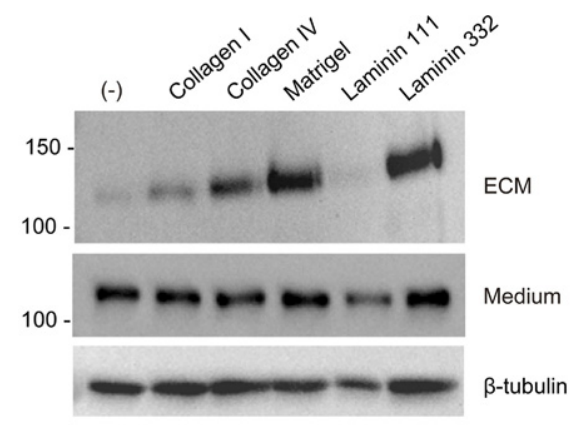

B
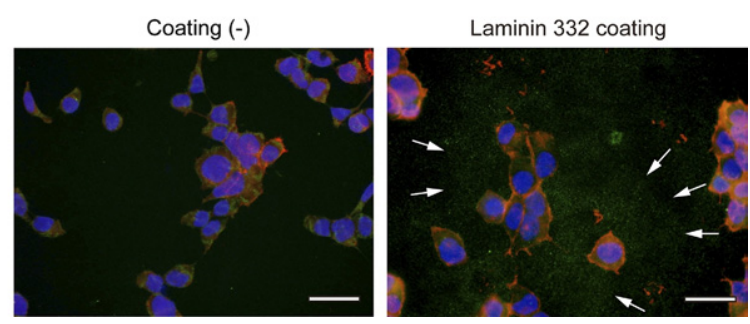

Figure 4. Deposition of Ecto-ColXVII in the ECM is enhanced by specific ligands. A: Immunoblot of the ECM and culture medium. The top panel shows that collagen XVII expressing 293 cells cultured on uncoated plastic (-) deposited negligible amounts of the Ecto-ColXVII in the ECM. The deposition was significantly increased when the cells were cultured on collagen IV, Matrigel, and laminin 332 but not on laminin 111. The Ab HK139 was used to detect the shed Ecto-ColXVII in the ECM. The middle panel shows the shed Ecto-ColXVII in culture medium, as detected by the $\mathrm{Ab}$ NC16A-3. The bottom panel shows $\beta$-tubulin in cell lysates. B: Consistent with the previous data on immunoblotting, IIF with the Ab HK139 detected shed Ecto-ColXVII (green; arrows) around cells cultured on laminin 332 coating. Red, rhodamine phalloidin; blue, DAPI. Scale bars $=40 \mu \mathrm{m}$. proteins, among which collagen IV and laminin 111 are major components. ${ }^{29}$ In the context of the present study, it is of interest whether Matrigel also contains laminin 332. Microarray data on Engelbreth-HolmSwarm tumor cells ${ }^{30}$ suggest that laminin 111, not laminin 332, is the major laminin in Matrigel. Accordingly, immunoblotting with a polyclonal laminin $332 \mathrm{Ab}$ did not show positive signals with Matrigel (data not shown). Culture on collagen IV moderately increased deposition of Ecto-CoIXVII, but laminin 111 failed to generate ECM binding. Culture on laminin 332 enhanced deposition of Ecto-CoIXVII strongly (Figure $4 \mathrm{~A}$ ), and, in agreement with the immunoblotting data, IIF with the Ab HK139 revealed increased amounts of Ecto-CoIXVII in the environment of cells seeded on laminin 332 (Figure 4B). These observations further corroborate the assumption that laminin 332 is a major binding partner of the Ecto-CoIXVII, next to collagen IV and/or other components in Matrigel, which can also bind the ectodomain. However, laminin 111 is not one of these.

\section{The C Terminus of the Ecto-ColXVII Is Essential for Interactions with the ECM}

To investigate the Ecto-ColXVII interaction with ECM proteins in more detail, we used migrating HEK 293 cells expressing five different deletion mutants of collagen $\mathrm{XVII}$ (Figure 5A). The ectodomains of all the mutants were detected in culture medium and had the expected molecular weight (Figure 5B), indicating that the mutants were correctly expressed and the ectodomains were shed from the cell surface. IIF and immunoblotting demonstrated that the ectodomains of full-length COL17 and of the deletion mutants designated as COL3 and NC6 were incorporated into the ECM but the shorter ectodomains of COL11 and COL 15 were not (Figure 5, $\mathrm{B}$ and $\mathrm{C}$ ). These data conclude that the region spanning Ser ${ }^{978}-$ Pro $^{1497}$ is necessary for collagen XVII to bind with the ECM.

Based on the previous observations, the amino acid stretch Ser ${ }^{978}-$ Arg $^{1174}$ in the ectodomain seemed to be important for ECM binding. To address this, a deletion construct of $\Delta$ Pro $^{997}$-Gly ${ }^{1152}$ was produced in which most of the candidate region was eliminated (Figure 5A). However, the ectodomain of this deleted mutant was efficiently shed from the cell surface and was incorporated into the ECM (Figure 5B), suggesting that different sequences and/or conformational factors in the Ser ${ }^{978}$. Pro ${ }^{1497}$ stretch are important for the interaction of collagen XVII with the ECM.

To define the binding regions of collagen XVII with different ECM proteins, HEK 293 cells stably expressing deletion mutants COL3, NC6, or $\triangle$ Pro $^{997}$-Gly ${ }^{1152}$ were cultured on different ECM proteins, and the ECM and the medium of these cultures were immunoblotted. The $A b$ HK139 demonstrated that coating with laminin 332 increased binding of the ectodomains of all the mutants (Figure 6A). Increased binding to collagen IV was observed with mutant NC6 but not with COL3, so binding of 
A

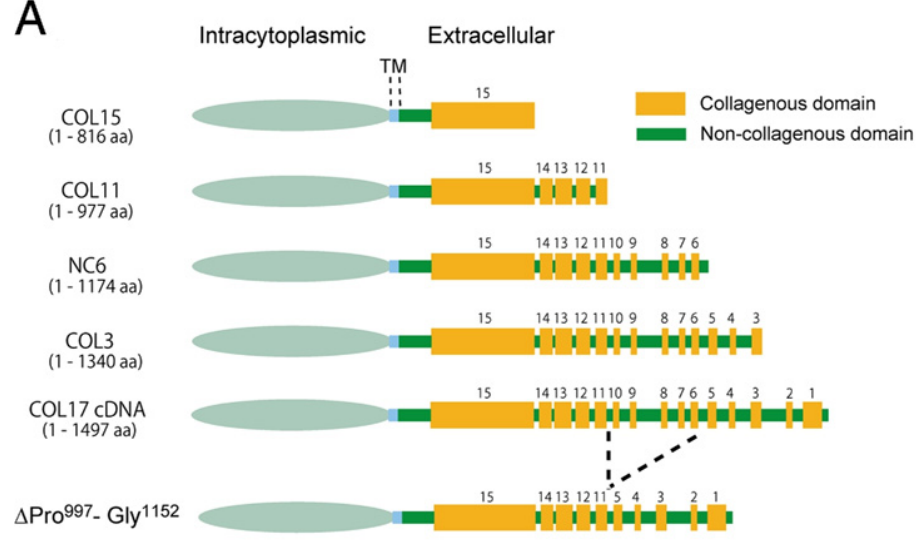

$\mathrm{B}$

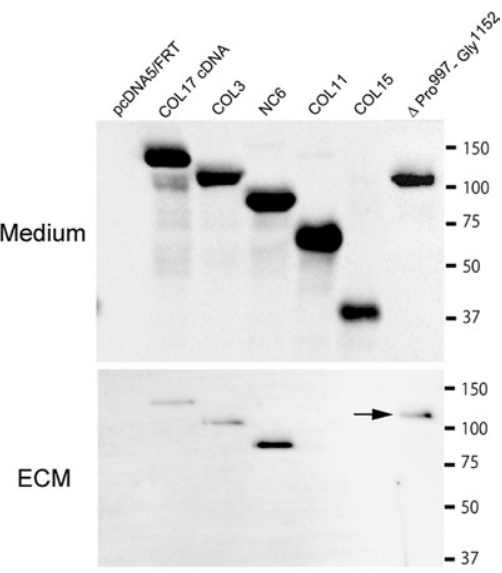

C
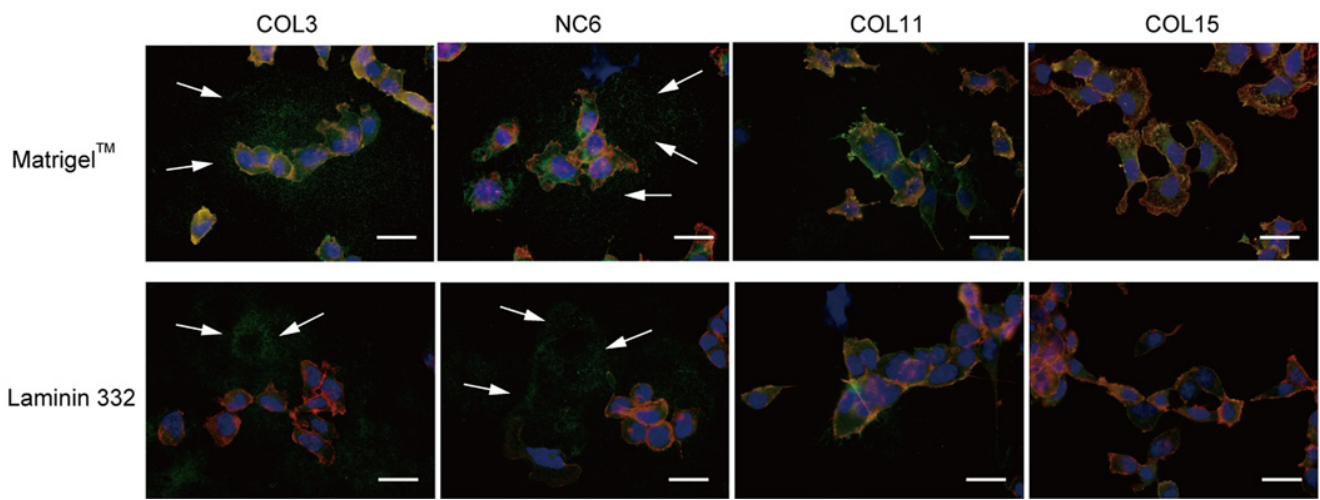

Figure 5. The C-terminal part of Ecto-ColXVII ( $\mathrm{Ser}^{978}-\mathrm{PrO}^{1497}$ ) is essential for ECM interactions. A: Schematic structure of the collagen XVII deletion mutants used in this study. B: The shed ectodomains of all the mutants were detected by the Ab NC16A-3 in the culture medium of transfected HEK 293 cells seeded on Matrigel. The molecular weight of the truncated ectodomains corresponded to the calculated sizes. Immunoblotting (B) and IIF (C) with the Ab HK139 demonstrated that the longer ectodomains of mutants COL3 and NC6 were deposited in the ECM (C; arrows) but that those of mutants COL11 and COL15 were not, indicating that the region spanning amino acids Ser ${ }^{978}$ to Pro ${ }^{1497}$ is necessary for binding of collagen XVII to the ECM. The mutant $\Delta$ Pro $^{997}$-Gly ${ }^{152}$ with an internal deletion also binds to the ECM $(\mathbf{B}$; arrow). TM, transmembrane; aa, amino acid; red, rhodamine phalloidin; blue, DAPI; and green, the Ab HK139 in $\mathbf{C}$. Scale bars $=40 \mu \mathrm{m}$.

collagen XVII with collagen IV was thought to be mediated mainly by the carboxyl terminal stretch $I l e^{1341}$. Pro ${ }^{1497}$. This is congruent with the result of full-length collagen XVII cDNA (Figure 4A). Increased binding to Matrigel was observed with COL3 and NC6 but not with the mutant $\Delta$ Pro $^{997}$-Gly ${ }^{1152}$ (Figure 6A), suggesting that the region Pro ${ }^{997}$-Gly ${ }^{1152}$ was important for the interaction of collagen XVII with Matrigel. Collagen IV, a major molecule in Matrigel, did not significantly increase binding of the mutant $\Delta$ Pro $^{997}$-Gly ${ }^{1152}$ (Figure $6 \mathrm{~A}$ ), indicating
A

A

$(-)$

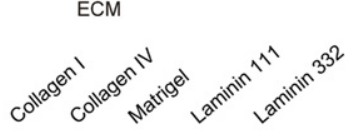

$\mathrm{COL} 3$
$(1-1340$ aa

NC6
$(1-1174$ aa $)$

$100-$

100 .

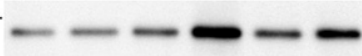

$\Delta$ Pro997- Gly1152

B

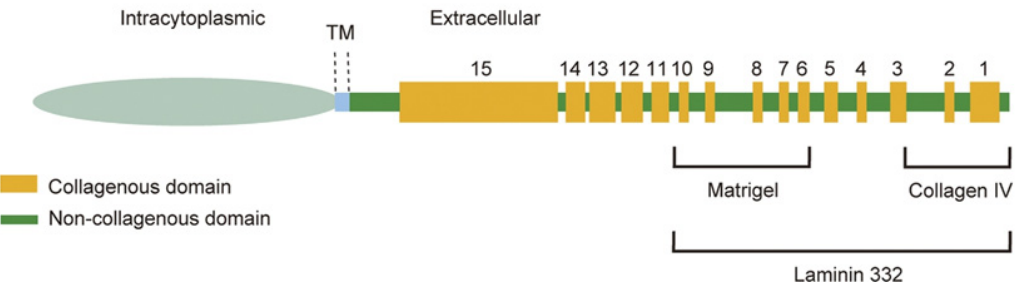

Medium

$(-)$

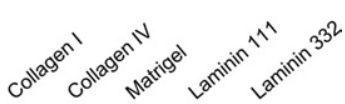

$100-$

100

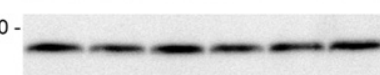

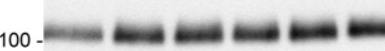

Figure 6. Differential interactions of Ecto-COLXVII with the ECM. The integration of the ectodomain into the matrix depends on the culture substrate. A: Immunoblot of the ECM and culture medium of cells expressing the deletion mutants COL3, NC6 and $\triangle$ Pro $^{997}$-Gly ${ }^{1152}$ cultured on different ECM proteins. Ecto-COLXVII was detected in the ECM by the Ab HK139 and in culture medium by the $\mathrm{Ab}$ NC16A-3. Coating with laminin 332 increased binding of the ectodomain of all mutants. In contrast, increased binding to collagen IV was observed only with $\Delta$ Pro $^{997}$-Gly ${ }^{1152}$. This, together with the result shown in Figure $4 \mathrm{~A}$, indicates that binding of collagen XVII to collagen IV is mainly mediated by the carboxyl terminus stretching from Ile $^{1341}$ to Pro ${ }^{1497}$. On Matrigel, incorporation of the ectodomain of $\Delta$ Pro $^{997}$-Gly ${ }^{1152}$ into the ECM was significantly lower than was that of the other mutants, suggesting that the limited region of $\mathrm{PrO}^{997}$ to Gly ${ }^{1152}$ is important for binding of collagen XVII to Matrigel. B: Schematic representation of putative interaction sites in the Ecto-ColXVII with different ECM proteins. aa, amino acid; TM, transmembrane. 
that in Matrigel, supramolecular assemblies and conformational determinants in collagen polymers create binding sites not present in a solution of single molecules used for coating of the cell culture surfaces in the present study. Alternatively, some hitherto unknown components may exist in Matrigel that are important for binding of the mutant Pro ${ }^{997}$ to Gly ${ }^{1152}$. Taken together, these observations suggest that although different ECM proteins bind to distinct regions of collagen XVII, laminin 332 is the major player (Figure 6B).

\section{Lack of Laminin 332 Affects Collagen XVII Deposition Into the ECM Differently in Vitro and in Vivo}

The experiments described previously herein disclosed a close relationship between collagen XVII and laminin 332 in the ECM. To assess whether laminin 332 is a necessary requirement for incorporation of collagen XVII into the ECM, we cultured keratinocytes from the skin of a patient with homozygous $L A M B 3$ mutation p.Arg635X and lack of laminin 332 expression, as shown by IIF staining of the skin of the patient with Abs to laminin $\alpha 3, \beta 3$, and $\gamma 2$ chains (data not shown). IIF staining with the Ab HK139 revealed that deposition of Ecto-CoIXVII in the ECM of laminin 332-negative keratinocytes was drastically reduced (Figure 7A) compared with that in the ECM of NHKs (Figure 2A). In contrast, in the skin of the same patient, moderate amounts of Ecto-ColXVII were present (Figure 7B). The total amount of collagen XVII was assayed with the monoclonal Ab NC16A-1, which recognizes the full-length and shed forms (Figure 7B). ${ }^{24}$ These observations indicate that in vitro, laminin 332 is essential for incorporation of collagen XVII into the ECM. However, in vivo, in the absence of laminin 332, other components will enter ligand interactions with the Ecto-ColXVII and retain it in the ECM in the skin.

A

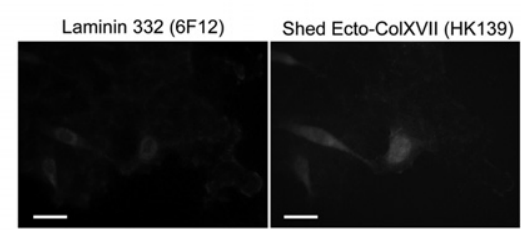

B

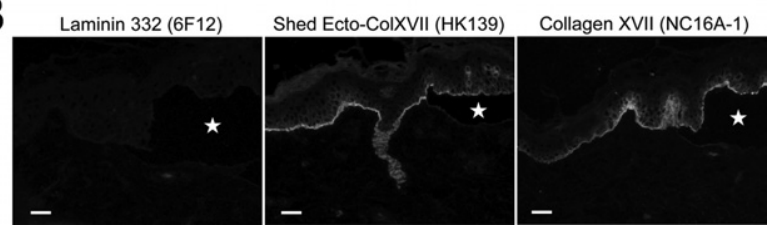

Figure 7. IIF of primary keratinocytes derived from a patient lacking laminin 332 expression. A: Staining with the $\mathrm{Ab} 6 \mathrm{~F} 12$ revealed a complete lack of laminin 332, and staining with the Ab HK139 strongly reduced amounts of shed Ecto-ColXVII in the ECM. B: However, in the skin of the same patient, moderate amounts of shed Ecto-ColXVII were seen (the Ab HK139). The monoclonal Ab NC16A-1, which recognizes the shed and full-length forms of collagen XVII, also generated a positive signal. These results indicate that in vitro laminin 332 is pivotal for incorporation of Ecto-ColXVII into the ECM, whereas other proteins will enter ligand interactions with the shed EctoColXVII and retain it in the ECM regardless of laminin 332 expression in vivo. Stars indicate subepidermal blister formations. Scale bars $=40 \mu \mathrm{m}$.

\section{Discussion}

In this study, we demonstrate that shedding of the EctoCoIXVII occurs dynamically in the ECM of migrating NHKs and that deposition of the Ecto-CoIXVII in the matrix requires ligand interactions, preferably with laminin 332, a major basement membrane protein in the skin.

Based on earlier binding studies using prokaryotic protein fragments, we suggested interactions between collagen XVII and laminin 332. ${ }^{19}$ Other investigators had found integrin $\alpha 6 \beta 4$ and collagen IV, in addition to laminin 332, in NHK migration tracks. ${ }^{31}$ However, lack of specific Abs recognizing only the shed Ecto-CoIXVII has hampered verification of the presence of the ectodomain in the ECM of cell cultures or tissues. Recent availability of the ectodomainspecific Ab HK139 ${ }^{17}$ now allowed us to analyze shedding of collagen XVII and deposition of the Ecto-CoIXVII into the ECM under physiologic and pathologic conditions.

We used three relevant cell systems: primary NHKs derived from human skin and the HaCaT keratinocyte cell line, which constitutively synthesize collagen XVII. Transfected HEK 293 epithelial cells were used to express recombinant mutant forms of collagen XVII. The findings corroborate the hypothesis of collagen XVII-laminin 332 interactions. Most often, the shed Ecto-ColXVII was co-localized with laminin 332 in the ECM of migrating NHKs. However, careful observation revealed that some migrating $\mathrm{NHKs}$ left tracks containing large amounts of laminin 332 but very little or no shed Ecto-ColXVII, which suggests that not all NHKs have the same pattern of migration and ECM deposition. This may reflect the biologic context of the cell at that particular moment. For example, our experiments showed that an increase in extracellular calcium concentration not only induces keratinocyte differentiation but may also influence expression and shedding of collagen XVII.

Little is known about other binding partners of EctoCoIXVII in the ECM. An in vitro study had suggested $\alpha 6$ integrin to be a ligand of the ectodomain, the binding site being within the juxtamembranous NC16A domain, specifically within the stretch spanning $\mathrm{Arg}^{506}-\mathrm{Il} \mathrm{e}^{519} .{ }^{16}$ We recently disclosed the major sheddase cleavage sites in the NC16A domain and the physiologic N-termini of the shed ectodomain. ${ }^{17}$ All these are located C-terminally from the putative $\alpha 6$ integrin binding site. Thus, the integrin binding site is not part of the shed Ecto-CoIXVII, and $\alpha 6$ integrin-collagen $\mathrm{XVII}$ interactions may rather regulate cellular events than be relevant for ECM binding.

To identify the domains of collagen XVII that are required for ECM interactions, we used HEK 293 cells transformed to stably express human collagen XVII and its deletion mutants. HEK 293 cells expressing full-length human collagen XVII only deposit minor amounts of ECM when cultured on plastic. However, when the cells were cultured on surfaces coated with laminin 332, collagen IV, or Matrigel, shed Ecto-CoIXVII was found in the ECM of the migration tracks. Laminin 111, a major component of Matrigel, ${ }^{29}$ did not support deposition of EctoColXVII at all. Thus, these findings support the important role of distinct ECM proteins for the incorporation of collagen XVII into the epidermal basement membrane. Support for these findings was found in a recent 
study that assessed cell adhesion to ECM using recombinant expression of collagen XVII. ${ }^{32}$ However, that study did not consider the role of the shed EctoCoIXVII and its interactions with the ECM.

To define the vital ECM binding sites in the Ecto-CoIXVII, deletion mutants were generated and stably expressed in HEK 293 cells. The experiments showed that a rather long stretch, Ser ${ }^{978}$-Pro ${ }^{1497}$, was necessary for binding with the ECM. It seems that different regions in the ectodomain engage in binding to distinct ECM molecules. The most carboxyl terminal stretch, $\| e^{1341}$ to Pro $^{1497}$, mediates binding to collagen IV, whereas a longer portion of the distal ectodomain, spanning from $\mathrm{Ser}^{978}$ to Pro $^{1497}$, is required for binding to laminin 332. In contrast, a limited region, Pro $^{997}$ to Gly ${ }^{1152}$, mediates interactions with Matrigel. These observations suggest that although laminin 332 is the major player, different ECM proteins can act as ligands for collagen XVII, presumably in a context-dependent manner. Previous studies with recombinant prokaryotic fragments had suggested that the most C-terminal part of collagen XVII, spanning collagenous domains 1 to 6 , was sufficient for laminin 332 binding. ${ }^{19}$ However, in the present study, use of epithelial cells in a more relevant in vitro setting showed that also other regions in the ectodomain played a role in ECM binding, possibly through conformational factors. This observation does not contradict the earlier data because protein conformation and posttranslational modifications are known to be important prerequisites for protein-protein interactions, and prokaryotic fragments do not always undergo proper folding owing to lack of posttranslational modifications.

The in vitro studies suggested strong association of laminin 332 with the ECM and the shed Ecto-ColXVII. Consequently, laminin 332-negative primary keratinocytes derived from the skin of a patient with Herlitz junctional epidermolysis bullosa deposited less shed EctoColXVII into the ECM. However, in the skin of the same patient shed Ecto-CoIXVII was detected at the dermoepidermal basement membrane. This finding implicates other molecules that can bind the extracellular domain of collagen XVII in vivo. This notion is supported by several observations. First, the present study and other investigations showed that collagen IV is also a relevant ligand for the Ecto-CoIXVII. ${ }^{18}$ Second, when artificial blistering of human skin is induced by $1 \mathrm{~mol} / \mathrm{L} \mathrm{NaCl}$, collagen XVII separates to the epidermal side of the blister and not to the dermal side with laminin 332. ${ }^{33,34}$ Also, the Ab HK139 detected the shed Ecto-ColXVII on the epidermal side of 1 $\mathrm{mol} / \mathrm{L} \mathrm{NaCl}$ split skin, ${ }^{17}$ suggesting that yet other epidermisassociated molecules interact with the Ecto-CoIXVII. Future studies will elucidate these binding partners.

This study defined shedding of the Ecto-CoIXVII as a dynamic process in the ECM and laminin 332 as a key binding partner, in addition to other basement membrane components important for cell adhesion.

\section{Acknowledgments}

We thank Margit Schubert and Yuko Hayakawa for their technical assistance and Dr. Cristina Has, Dr. Ying-Hong
$\mathrm{He}$, and Daniela Velati for their help with the experiments and their critical suggestions.

\section{References}

1. Hynes RO: The extracellular matrix: not just pretty fibrils. Science 2009, 326:1216-1219

2. Heino J, Kapyla J: Cellular receptors of extracellular matrix molecules. Curr Pharm Des 2009, 15:1309-1317

3. Barczyk M, Carracedo S, Gullberg D: Integrins. Cell Tissue Res 2010, 339:269-280

4. Franzke CW, Bruckner P, Bruckner-Tuderman L: Collagenous transmembrane proteins: recent insights into biology and pathology. J Biol Chem 2005, 280:4005-4008

5. Sugawara K, Tsuruta D, Ishii M, Jones JC, Kobayashi H: Laminin-332 and -511 in skin. Exp Dermatol 2008, 17:473-480

6. Tsuruta D, Kobayashi H, Imanishi H, Sugawara K, Ishii M, Jones JC Laminin-332-integrin interaction: a target for cancer therapy? Curr Med Chem 2008, 15:1968-1975

7. McMillan JR, Akiyama M, Shimizu H: Epidermal basement membrane zone components: ultrastructural distribution and molecular interactions. J Dermatol Sci 2003, 31:169-177

8. Has C, Bruckner-Tuderman L: Molecular and diagnostic aspects of genetic skin fragility. J Dermatol Sci 2006, 44:129-144

9. Van Agtmael T, Bruckner-Tuderman L: Basement membranes and human disease. Cell Tissue Res 2010, 339:167-188

10. Pulkkinen L, Christiano AM, Gerecke D, Wagman DW, Burgeson RE, Pittelkow MR, Uitto J: A homozygous nonsense mutation in the $\beta 3$ chain gene of laminin 5 (LAMB3) in Herlitz junctional epidermolysis bullosa. Genomics 1994, 24:357-360

11. McGrath JA, Gatalica B, Christiano AM, Li K, Owaribe K, McMillan JR, Eady RA, Uitto J: Mutations in the 180-kD bullous pemphigoid antigen (BPAG2), a hemidesmosomal transmembrane collagen (COL17A1), in generalized atrophic benign epidermolysis bullosa. Nat Genet 1995, 11:83-86

12. Giudice GJ, Emery DJ, Diaz LA: Cloning and primary structural analysis of the bullous pemphigoid autoantigen BP180. J Invest Dermatol 1992, 99:243-250

13. Li K, Tamai K, Tan EM, Uitto J: Cloning of type XVII collagen: complementary and genomic DNA sequences of mouse 180-kilodalton bullous pemphigoid antigen (BPAG2) predict an interrupted collagenous domain, a transmembrane segment, and unusual features in the $5^{\prime}$ end of the gene and the 3' untranslated region of the mRNA. J Biol Chem 1993, 268:8825-8834

14. Schaapveld RQ, Borradori L, Geerts D, van Leusden MR, Kuikman I, Nievers MG, Niessen CM, Steenbergen RD, Snijders PJ, Sonnenberg A: Hemidesmosome formation is initiated by the $\beta 4$ integrin subunit, requires complex formation of $\beta 4$ and HD1/plectin, and involves a direct interaction between $\beta 4$ and the bullous pemphigoid antigen 180. J Cell Biol 1998, 142:271-284

15. Koster J, Geerts D, Favre B, Borradori L, Sonnenberg A: Analysis of the interactions between BP180. BP230, plectin and the integrin $\alpha 6 \beta 4 \mathrm{im}$ portant for hemidesmosome assembly. J Cell Sci 2003, 116:387-399

16. Hopkinson SB, Findlay K, deHart GW, Jones JC: Interaction of BP180 (type XVII collagen) and $\alpha 6$ integrin is necessary for stabilization of hemidesmosome structure. J Invest Dermatol 1998, 111:1015-1022

17. Nishie W, Lamer S, Schlosser A, Licarete E, Franzke CW, Hofmann SC, Jackow J, Sitaru C, Bruckner-Tuderman L: Ectodomain shedding generates Neoepitopes on collagen XVII, the major autoantigen for bullous pemphigoid. J Immunol 2010, 185:4938-4947

18. Qiao H, Shibaki A, Long HA, Wang G, Li Q, Nishie W, Abe R, Akiyama M, Shimizu H, McMillan JR: Collagen XVII participates in keratinocyte adhesion to collagen IV, and in p38MAPK-dependent migration and cell signaling. J Invest Dermatol 2009, 129:2288-2295

19. Tasanen K, Tunggal L, Chometon G, Bruckner-Tuderman L, Aumailley M: Keratinocytes from patients lacking collagen XVII display a migratory phenotype. Am J Pathol 2004, 164:2027-2038

20. Parikka M, Nissinen L, Kainulainen T, Bruckner-Tuderman L, Salo T, Heino J, Tasanen K: Collagen XVII promotes integrin-mediated squamous cell carcinoma transmigration: a novel role for $\alpha \mathrm{llb}$ integrin and tirofiban. Exp Cell Res 2006, 312:1431-1438 
21. Pulkkinen L, Gerecke DR, Christiano AM, Wagman DW, Burgeson RE, Uitto J: Cloning of the $\beta 3$ chain gene (LAMB3) of human laminin 5 , a candidate gene in junctional epidermolysis bullosa. Genomics 1995 25:192-198

22. Franzke CW, Tasanen K, Schacke H, Zhou Z, Tryggvason K, Mauch C Zigrino P, Sunnarborg S, Lee DC, Fahrenholz F, Bruckner-Tuderman L: Transmembrane collagen XVII, an epithelial adhesion protein, is shed from the cell surface by ADAMs. EMBO J 2002, 21:5026-5035

23. Franzke CW, Tasanen K, Borradori L, Huotari V, Bruckner-Tuderman L: Shedding of collagen XVII/BP180: structural motifs influence cleavage from cell surface. J Biol Chem 2004, 279:24521-24529

24. Hofmann SC, Voith U, Schonau V, Sorokin L, Bruckner-Tuderman $\mathrm{L}$, Franzke CW: Plasmin plays a role in the in vitro generation of the linear IgA dermatosis antigen LADB97. J Invest Dermatol 2009, 129:1730-1739

25. Marinkovich MP, Lunstrum GP, Burgeson RE: The anchoring filament protein kalinin is synthesized and secreted as a high molecular weight precursor. J Biol Chem 1992, 267:17900-17906

26. Langhofer M, Hopkinson SB, Jones JC: The matrix secreted by $804 \mathrm{G}$ cells contains laminin-related components that participate in hemidesmosome assembly in vitro. J Cell Sci 1993, 105 (Pt 3):753-764

27. Stanley JR, Yuspa SH: Specific epidermal protein markers are modulated during calcium-induced terminal differentiation. J Cell Biol 1983, 96:1809-1814
28. Franzke CW, Bruckner-Tuderman L, Blobel CP: Shedding of collagen XVII/BP180 in skin depends on both ADAM10 and ADAM9. J Biol Chem 2009, 284:23386-23396

29. Kleinman HK: Preparation of basement membrane components from EHS tumors. Curr Protoc Cell Biol 2001, 10.210:

30. Futaki S, Hayashi Y, Yamashita M, Yagi K, Bono H, Hayashizaki Y, Okazaki Y, Sekiguchi K: Molecular basis of constitutive production of basement membrane components: gene expression profiles of Engelbreth-Holm-Swarm tumor and F9 embryonal carcinoma cells. J Biol Chem 2003, 278:50691-50701

31. Kirfel G, Rigort A, Borm B, Schulte C, Herzog V: Structural and compositional analysis of the keratinocyte migration track. Cell Moti Cytoskeleton 2003, 55:1-13

32. Van den Bergh F, Eliason SL, Giudice GJ: Type XVII collagen (BP180) can function as a cell-matrix adhesion molecule via binding to laminin 332. Matrix Biol 2011, 30:100-108

33. Lazarova Z, Yancey KB: Reactivity of autoantibodies from patients with defined subepidermal bullous diseases against $1 \mathrm{~mol} / \mathrm{L}$ salt-split skin: specificity, sensitivity, and practical considerations. J Am Acad Dermatol 1996, 35:398-403

34. Murakami H, Nishioka S, Setterfield J, Bhogal BS, Black MM, Zillikens D, Yancey KB, Balding SD, Giudice GJ, Diaz LA, Nishikawa T, Kiyokawa $\mathrm{C}$, Hashimoto $\mathrm{T}$ : Analysis of antigens targeted by circulating $\lg \mathrm{G}$ and IgA autoantibodies in 50 patients with cicatricial pemphigoid. J Dermatol Sci 1998, 17:39-44 